\title{
Mitral valve leaflets abscess
}

\author{
Grzegorz Styczynski ${ }^{1}$, Cezary Szmigielski ${ }^{1}$, and Joanna Bidiuk ${ }^{1}$ \\ ${ }^{1}$ Medical University of Warsaw
}

June 1, 2020

\begin{abstract}
Mitral valve abscess is a well-known complication of infective endocarditis, often requiring surgical treatment. Usually, the most typical localization is postero-lateral mitral annulus or mitral-aortic curtain at the base of the anterior leaflet in aortic valve endocarditis. We describe a 64-year-old male diagnosed with the abscess on the free margins of the mitral leaflets secondary to concomitant vertebral osteomyelitis. A favorable resolution of the abscess during the intravenous dual antibiotic therapy is presented.
\end{abstract}

Mitral valve leaflets abscess

Styczyński Grzegorz, MD, PhD, Medical University of Warsaw

Szmigielski Cezary, MD, PhD, Medical University of Warsaw

Bidiuk Joanna, MD, Medical University of Warsaw, Department of Internal Medicine, Hypertension and Vascular Diseases, UCK WUM, 1A Banacha Street, 02-097 Warsaw, Poland. Phone +48 2259928 28, Fax +48 2259918 28. Email: jbidiuk@wum.edu.pl

Authors:

Styczyński Grzegorz, MD, PhD, Department of Internal Medicine, Hypertension and Vascular Diseases, Medical University of Warsaw

Szmigielski Cezary, MD, PhD, Department of Internal Medicine, Hypertension and Vascular Diseases, Medical University of Warsaw

Bidiuk Joanna, MD, Department of Internal Medicine, Hypertension and Vascular Diseases, Medical University of Warsaw

Corresponding author:

Bidiuk Joanna, Department of Internal Medicine, Hypertension and Vascular Diseases, UCK WUM, 1A Banacha Street, 02-097 Warsaw, Poland. Phone +48 2259928 28, Fax +48 2259918 28. Email: jbidiuk@wum.edu.pl

Author contributions:

Styczyński Grzegorz: Concept and design, Data collection, Critical revision of article, Approval of article. Szmigielski Cezary: Design, Critical revision of article, Approval of article

Bidiuk Joanna: Drafting article, Approval of article.

Abstract

Mitral valve abscess is a well-known complication of infective endocarditis, often requiring surgical treatment. Usually, the most typical localization is postero-lateral mitral annulus or mitral-aortic curtain at the base of 
the anterior leaflet in aortic valve endocarditis. We describe a 64-year-old male diagnosed with the abscess on the free margins of the mitral leaflets secondary to concomitant vertebral osteomyelitis. A favorable resolution of the abscess during the intravenous dual antibiotic therapy is presented.

Keywords: echocardiography, infective endocarditis (IE), mitral valve, paravalvular abscess

Case presentation

64-years old male with the history of alcohol abuse was admitted to the hospital because of fever, weakness and back pain. During initial diagnostic evaluation, all three blood cultures were positive for methicillinsensitive Staphylococcus aureus and Streptococcus vestibularis. Further tests for suspected endocarditis involved transesophageal echocardiography (TEE), which showed a rounded, bi-lobal, hyperechogenic mass localized at the tips of the both, anterior and posterior mitral leaflets (Figure 1, panel A, B, C, Movie S1 and $\mathrm{S} 2$ ). Concurrently, mild to moderate mitral insufficiency was present. No signs of heart failure or systemic embolism were identified. Intravenous antibiotics (ceftriaxone and gentamycin) were started according to the guidelines (1). Subsequent diagnostic work-up for the source of bacteremia, revealed additionally, a presence of the L1 vertebral osteomyelitis, with adjacent right psoas muscle abscess. According to the cardiosurgical, orthopedic and neurosurgical consultations, a conservative treatment with intravenous antibiotics was recommended to be continued. A follow-up TEE performed one week later demonstrated the disruption of the mitral mass with decrease in its size and the occurrence of single, highly mobile structure, consistent with bacterial vegetation on the tip of the mitral leaflet (Figure 1, panel D,E,F, Movie S3 and S4 ). During the follow up. there was no increase in the degree of mitral insufficiency, nor clinical signs of heart failure or embolism. Another TEE performed after one week (i.e. 2 weeks after initiation of antibiotics), revealed an almost complete resolution of the mitral mass, without any significant increase in mitral insufficiency, including no signs of perforation of the mitral leaflets (Figure 1, panel G,H,I, Movie S5 and S6). However, at the same time, progressive vertebral destruction led to localized spinal cord compression requiring subsequent neurosurgical treatment.

Discussion

Prevalence of infective endocarditis (IE) in patients initially diagnosed with infective spondylodiscitis, and systematically evaluated with TEE is high, reaching up to 30 percent, and is associated with significantly worse prognosis (2). In this patient, an initial echocardiographic assessment (normal left ventricular and atrial size, mild central mitral regurgitation) suggested no evident valvular predisposition for infective endocarditis. This underscores the high risk for infection of normal native valves during persistent, significant bacteremia from distant sites, like in our patient with infective spondylodiscitis (1). It is unclear if polymicrobial character of bacteriemia in this patient, contributed to the increased risk of endocardial involvement. However, based on the data from the literature, polymicrobial bacteremia with the presence of Staphylococcus aureus and variety of clinical manifestations, is generally associated with worse prognosis and higher mortality, compared to monomicrobial staphylococcal bacteremia only $(3,4)$. Concluding, mitral valve abscess is a well-known and severe complication of infective endocarditis, potentially life-threatening, and often requiring surgical treatment. However, the most typical localization is postero-lateral mitral annulus or mitral-aortic curtain at the base of anterior leaflet in concomitant aortic valve endocarditis (1). In this case, we describe an infrequent, in our observation, example of an abscess on the free margins of the mitral leaflets, with its favorable resolution during intravenous treatment with two antibiotics. A superficial, nodular morphology of the abscess at initial TEE examinations may suggest its early stage of development, not yet complicated by any localized mitral leaflets destruction.

\section{References:}

1. Habib G, Lancellotti P, Antunes MJ, et al: 2015 ESC Guidelines for the management of infective endocarditis: The Task Force for the Management of Infective Endocarditis of the European Society of Cardiology (ESC). Eur Heart J. 2015;36):3075-3128.

2. Behmanesh B, Gessler F, Schnoes K, et al: Infective endocarditis in patients with pyogenic spondylodisci- 
tis: implications for diagnosis and therapy. Neurosurg Focus. 2019 Jan 1;46(1):E2.

3. Khatib R, Sharma M, Johnson L, et al: Polymicrobial Staphylococcus aureus bacteremia: Frequency, distinguishing characteristics and outcome. Diagnostic Microbiology and Infectious Disease 2016;86: 311315 .

4. Park S, Park K, Bang K, et al: Clinical significance and outcome of polymicrobial Staphylococcus aureus bacteremia. Journal of Infection 2012; 65:119-127.

Figure 1. Panel A - 2D TEE, mid-esophageal long axis view at $124^{\circ}$ angle rotation, panel B- 2D TEE, mid esophageal commissural view at $52^{\circ}$ angle rotation, panel C- 3D TEE modified atrial view. Thick arrows point to mitral leaflets abscess. Panels D, E, F - respective views of the TEE study performed 1 weeks later. Thin arrows point to the vegetation. Panels G, H, I - respective views of the TEE study performed 2 weeks later.

Supplementary Material: The following supplementary material is available online - Movie Clips:

Movie S1: 2D TEE, mid-esophageal long axis view at $124^{\circ}$ angle rotation, Movie S2: 2D TEE, mid esophageal commissural view at $52^{\circ}$ angle rotation, showing rounded, bi-lobal, hyperechogenic masses localized at the tips of the anterior and posterior mitral leaflets

Movie S3: 2D TEE, mid-esophageal long axis view at $138^{\circ}$ angle rotation, Movie S4: 2D TEE, mid esophageal commissural view at $74^{\circ}$ angle rotation, showing disruption of the mitral mass with decrease in its volume and the presence of single, highly mobile structure consistent with vegetation.

Movie S5: 2D TEE, mid-esophageal long axis view at $129^{\circ}$ angle rotation, performed 2 weeks later, Movie S6: $2 \mathrm{D}$ TEE, mid esophageal commissural view at $49^{\circ}$ angle rotation, performed 2 weeks later, showing nearly complete resolution of the mass.

Codec used: Microsoft Video 1 (CRAM)

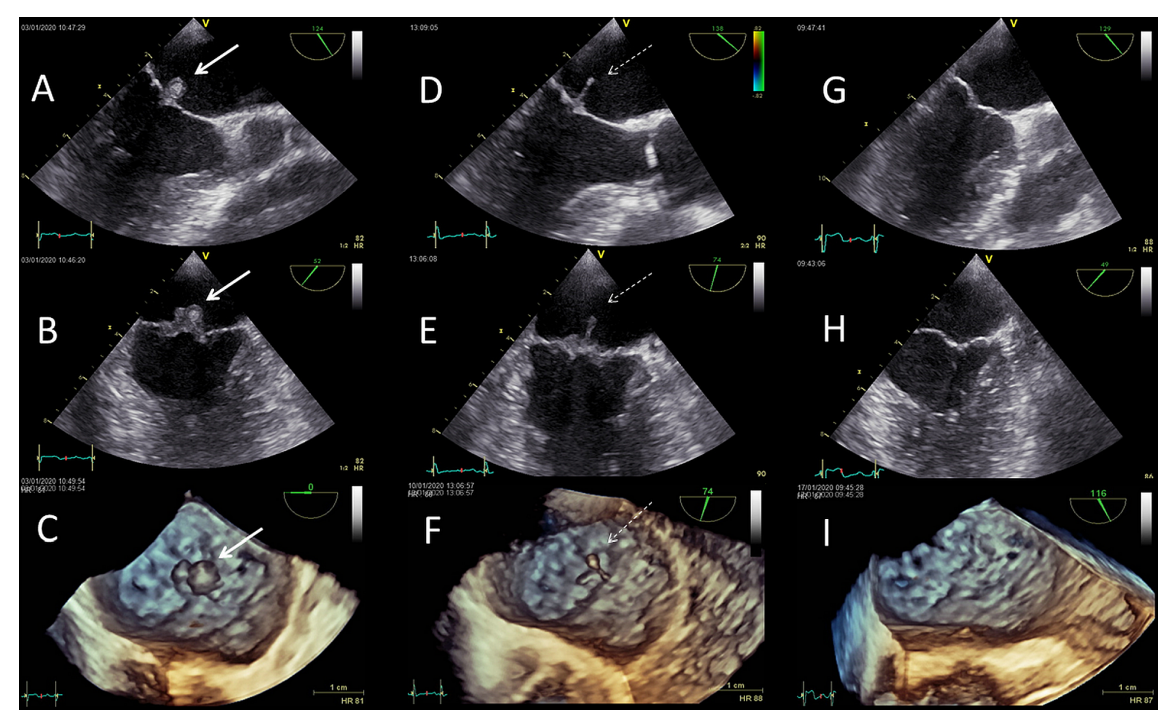

